\title{
Multi objective optimization model of railway transportation logistics network based on improved ant colony algorithm
}

\author{
LIU Xiu-miao \\ \{LiuxiuMiao22@163.com\} \\ HEBEI VOCATIONAL COLLEGE OF RAIL TRANSPORTATION, Shijiazhuang 050000, China
}

\begin{abstract}
In view of the problem that the transportation cost of the original multi objective optimization model of railway transportation logistics network is too high, this paper introduces the improved ant colony algorithm and designs the multi objective optimization model of railway transportation logistics network based on the improved ant colony algorithm. Set the logistics warehouse as the logistics transportation node and calculate the node optimization cost; use the improved ant colony algorithm to obtain the optimal transportation path in the railway transportation logistics network; set the optimal path as the form of particles, and use the particle swarm algorithm to complete the multi objective optimal solution process of the railway transportation logistics network, and build the multi objective optimization model. So far, the multi objective optimization model design of railway transportation logistics network based on improved ant colony algorithm is completed. Compared with the original optimization model, the application effect of this optimization model is better, which can effectively reduce the cost of logistics network transportation.
\end{abstract}

Keywords: improved ant colony algorithm; multi objective optimization; railway transportation; logistics network;

\section{Introduction}

Railway transportation is a basic industry related to the national economy and people's livelihood. Its security, security, coverage and advanced technology play a vital role in our daily life and the country's basic economy. [1] In recent years, the pressure and challenges faced by the railway transportation industry are more and more serious. Therefore, the railway logistics company must strengthen its service capacity, reduce costs and increase efficiency, so as to meet the requirements of social and economic development. With the development of logistics industry, railway logistics plays a more and more important role in the development of logistics industry. Reducing logistics cost and improving logistics service is a great 
opportunity for the development of railway logistics company. The service ability of logistics is based on the logistics network of railway transportation. Therefore, the optimization of the node layout of the logistics network of railway logistics is the basis and key to improve the service level of railway logistics. It can effectively reduce the logistics cost of logistics companies, improve the efficiency of logistics operation, and achieve the purpose of cost reduction and efficiency increase of logistics companies [2].

Based on the above background, aiming at the existing problems of railway logistics network node layout, this paper designs a multi objective optimization model of railway logistics network. Firstly, based on the existing hierarchical structure of railway logistics network nodes, the improved structure of "virtual warehouse + regional warehouse + turnover warehouse" is proposed, which provides the premise and foundation for further research; then, through the theoretical research of warehouse location and regional aggregation, the regional aggregation model and cost model suitable for the optimization of railway logistics network node layout are proposed, aiming at the optimization of railway logistics network nodes At the same time, it determines the optimization of the regional warehouse, and analyzes the storage cost, transportation cost and service penalty cost to realize the optimization of the total cost of the railway logistics network, and determines the optimization of the turnover warehouse; then, it uses the modified ant colony algorithm to program the model; finally, it uses the experimental demonstration analysis method By analyzing the optimization results, the feasibility and effectiveness of the optimization method of railway logistics network node layout proposed in this paper are verified, which provides a new idea and method for optimizing the node layout of railway logistics network.

\section{Multi objective optimization model design of railway transportation logistics network based on improved ant colony algorithm}

Through the study of the original railway transportation logistics network, it can be seen that the original railway transportation network multi objective model can not select the optimal logistics route, resulting in the problem of high logistics cost. In this model design, the original logistics network model is optimized by using the improved ant colony algorithm to reduce the cost of railway transportation logistics. In order to ensure the effectiveness of the design process, the construction process model is used to control the optimization model design process. The specific process is as follows. 


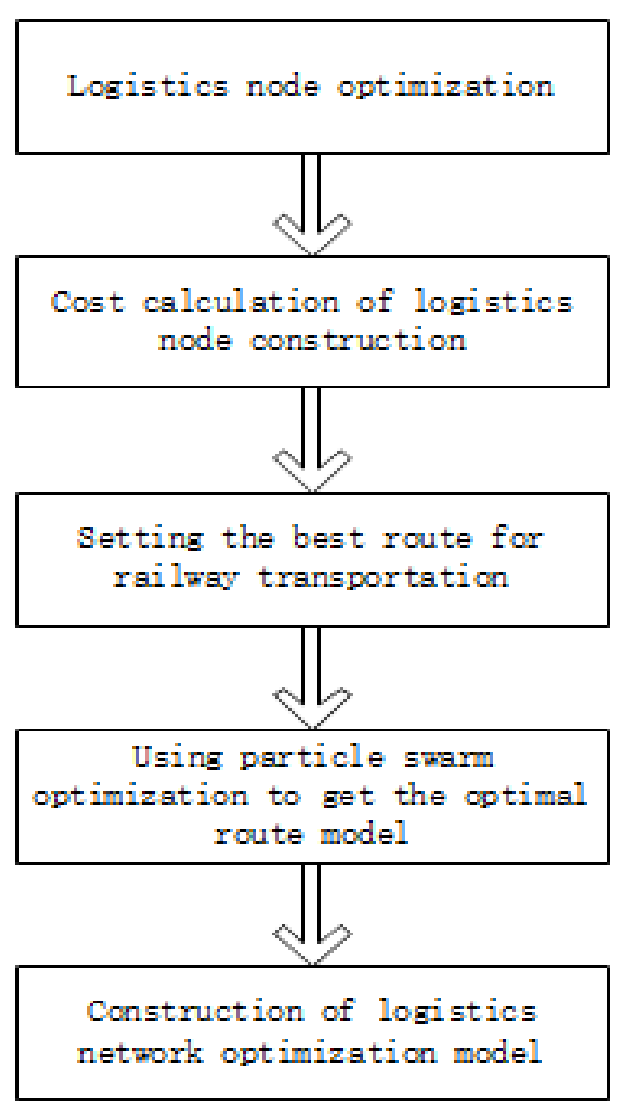

Fig. 1. Multi objective optimization model design flow of railway transportation logistics network based on improved ant colony algorithm

Use the above process to complete the design of the optimization model. In this design, in addition to improving the ant colony algorithm, particle swarm optimization is also added to ensure the accuracy and effectiveness of the optimization model design.

\subsection{Optimization of railway transportation logistics network nodes}

According to the related problems of the railway transportation logistics network proposed above, firstly, the hierarchical structure of the railway transportation logistics network node is adjusted, and the virtual warehouse in the logistics network is used as the information center of the railway logistics material warehouse, which is responsible for the overall management and coordination of the materials in the logistics network, so as to break the administrative barriers and improve the regional self-management of the material warehouse in the logistics network In order to achieve the top-down management goal of logistics network materials, we should give full play to the advantages of logistics standardization, intensification and scale 
[3-4], and further establish an integrated management system of "virtual warehouse + regional warehouse + turnover warehouse". The hierarchical structure of the optimized logistics network node is shown in the figure below.

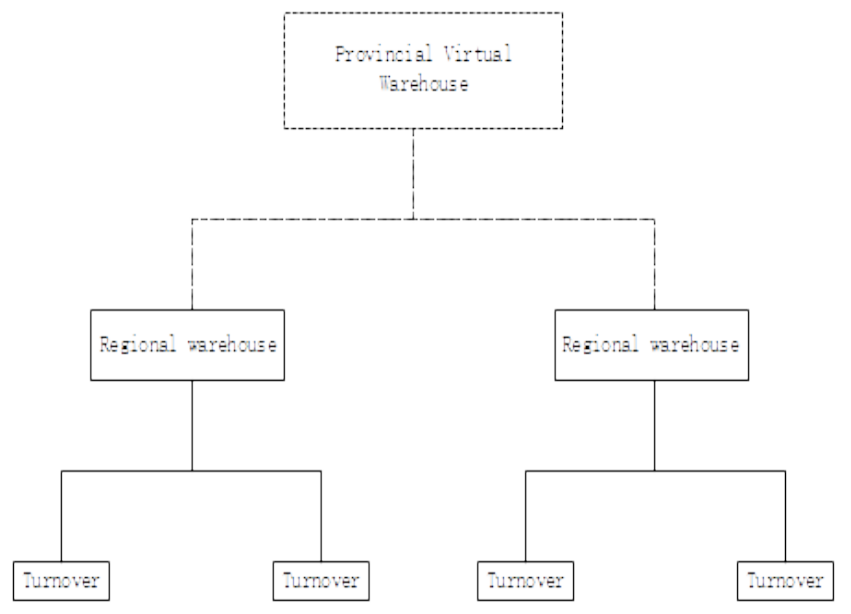

Fig. 2. Hierarchical structure of railway logistics network nodes

When optimizing the node layout of the logistics network, on the one hand, the service level of the optimized logistics network should be as high as possible, on the other hand, the lowest cost of the logistics network should be taken into account, so it is necessary to analyze the cost of the logistics network. In the previous section, the location of the regional warehouse of the logistics network is determined. Then, in the optimization of turnover warehouse, the location of regional warehouse is taken as the known condition, and the rest warehouses except the logistics network material warehouse which is selected as the regional warehouse are taken as the alternative warehouse of turnover warehouse to optimize the storage, transportation and service penalty cost [5], so as to determine the location of turnover warehouse. The cost parameter settings required by the network node are shown in the table below.

Table 1. Parameter setting for node cost

\begin{tabular}{lll}
\hline parameter & $\begin{array}{l}\text { Parameter } \\
\text { value }\end{array}$ & Meaning \\
\hline A & 3 & $\begin{array}{l}\text { Inventory turnover } \\
\text { Regional Library } \\
\text { B }\end{array}$ \\
& 4 & $\begin{array}{l}\text { Collection } \\
\text { Alternative node } \\
\text { set of turnover }\end{array}$
\end{tabular}




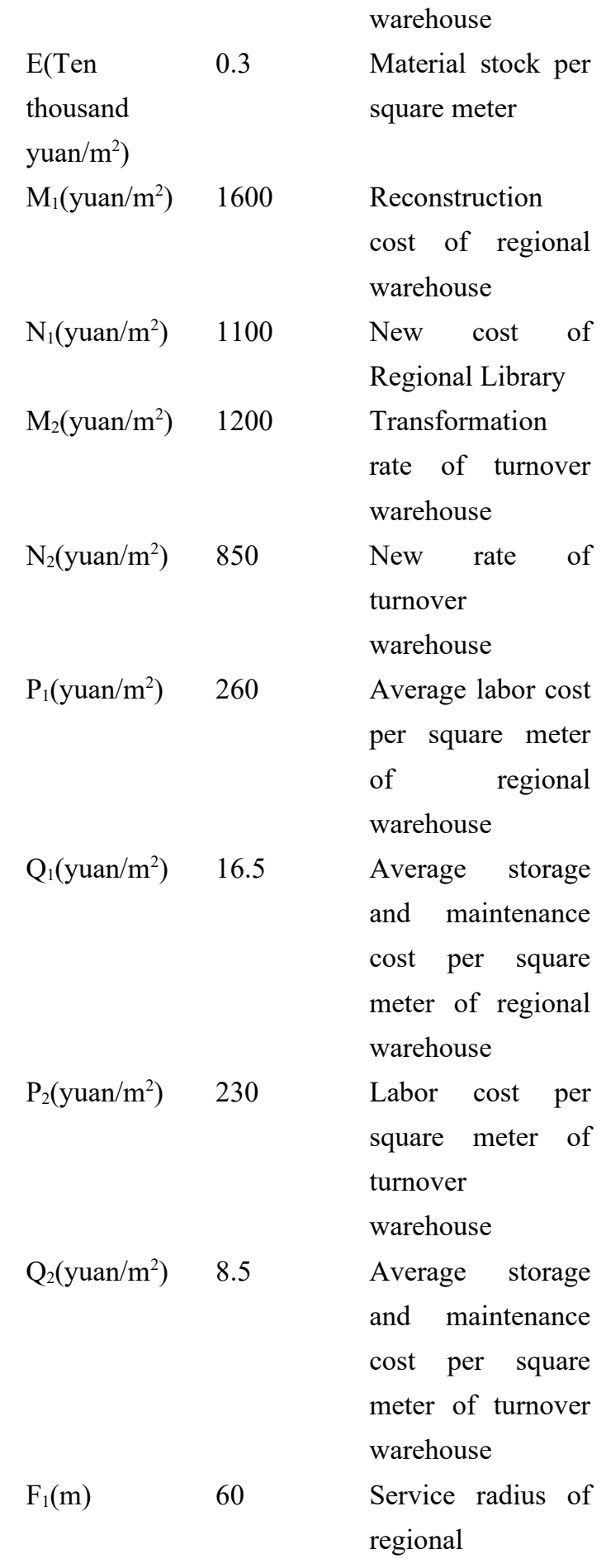




\begin{tabular}{|c|c|c|}
\hline & & warehouse \\
\hline $\mathrm{F}_{2}(\mathrm{~m})$ & 25 & $\begin{array}{l}\text { Service radius of } \\
\text { turnover } \\
\text { warehouse }\end{array}$ \\
\hline $\mathrm{T}_{1}($ yuan $/ \mathrm{m})$ & 13 & $\begin{array}{l}\text { Service radius of } \\
\text { turnover } \\
\text { warehouse }\end{array}$ \\
\hline $\mathrm{T}_{2}($ yuan $/ \mathrm{m})$ & 9 & $\begin{array}{l}\text { Service penalty } \\
\text { rate of turnover } \\
\text { warehouse }\end{array}$ \\
\hline
\end{tabular}

Through the above parameter setting, the cost of the optimized logistics network node is calculated, and the cost is scheduled as a constant value to ensure the optimized logistics network node, which can effectively reduce the logistics cost. The optimized nodes are taken as the design basis of the optimization model.

\subsection{Using improved ant colony algorithm to solve the optimal logistics path}

Using the above set logistics network nodes to complete the initialization work, combined with the characteristics of the original railway transport logistics, the optimal solution flow of the logistics path is formulated as follows. 


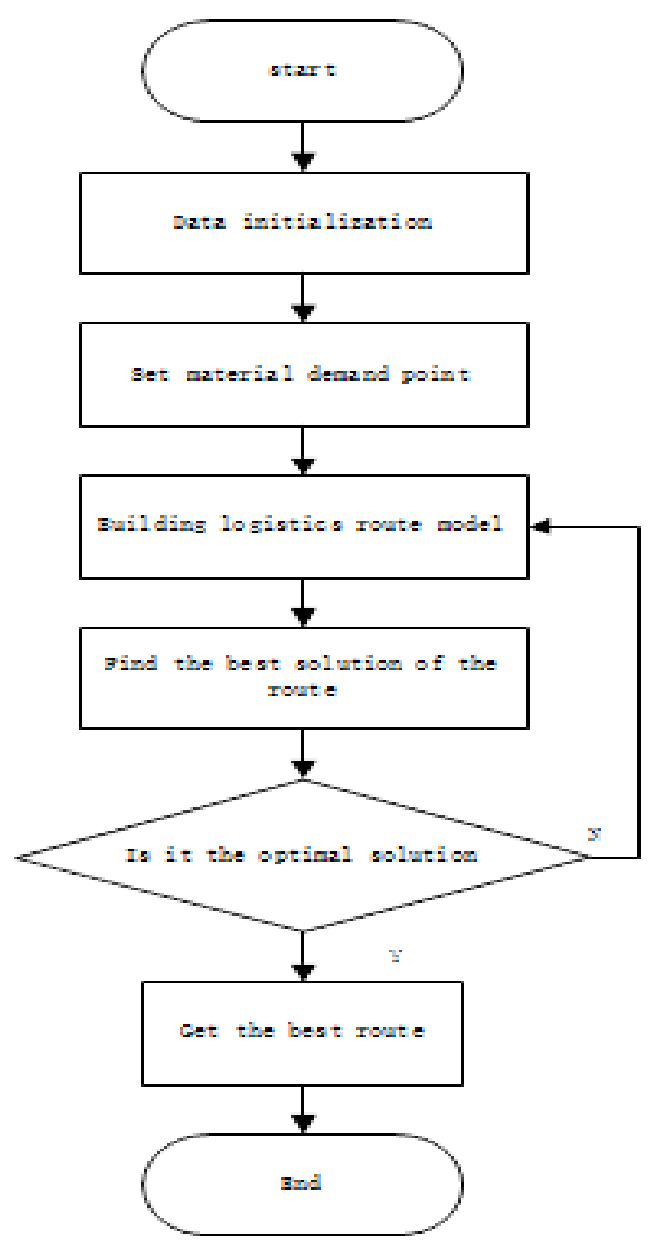

Fig. 3. Solution flow of the optimal route of logistics

According to the above process, ant colony algorithm is used to solve the optimal route [6-7]. Because ant colony algorithm is an evolutionary algorithm that simulates the behavior of ant colony in nature, it sets the logistics transport vehicle as an ant in the ant colony algorithm, and sets the feasible route from the material distribution center to the goods demand.

The action route of logistics vehicles is $a$, and the initial coordinate of the route is set as $\left[\left(m_{1}, n_{\text {start }}\right),\left(m_{n}, n_{\text {end }}\right)\right]$. In the route construction, there is a set of material demand points $W$, in which there are all the logistics points in the corresponding region, and these logistics points can meet the needs of railway transportation, logistics cost budget and transportation 
time. Select the appropriate material demand point in the set and insert it in front of $m_{n}$, and set it to connect with $m_{n}$. By implementing this step repeatedly, a new railway logistics transportation route can be obtained. In order to ensure that when the logistics transport vehicle goes to the next material demand point $f$ at a demand point $e$, variable $X$ is generated in the influence conditions of logistics transport. When $X \leq X_{0}$, the logistics vehicle will go to the next material demand point. The formula can be expressed as follows:

$$
\delta=\arg _{t \in T} \max \beta_{j} \mathfrak{J}_{j}
$$

In the above formula, $\beta_{j}, \mathfrak{J}_{j}$ are the influencing factors in the route advance, and $\delta$ is the information functions. When $X>X_{0}$, the logistics vehicle will go to the next material demand point. The formula can be expressed as follows:

$$
z_{m n}^{t}=\left\{\begin{array}{l}
\frac{\beta_{j} \mathfrak{J}_{j}}{\sum_{t \in T} \beta_{j} \mathfrak{I}_{j}}, t \in T \\
0, t \in T
\end{array}\right.
$$

Through the above formula, the order of material demand points can be set, and the obtained route is the current optimal solution. Combined with the above process cycle calculation, the correctness of the optimal solution can be verified.

\subsection{Reference multi objective fitness function to build multi objective optimization model of railway transportation logistics network}

Set the optimized route as the form of particles to complete the construction of the optimization model. After the path particles are encoded, their fitness can not get the optimal result in the program according to the objective function. It is necessary to adjust the fitness function [8-9] to make it optimal. The multi objective optimization problem is expressed as follows:

$$
y\left(r^{\prime}\right)=\left[y_{1}\left(r^{\prime}\right), y_{2}\left(r^{\prime}\right), \cdots, y_{n}\left(r^{\prime}\right)\right]
$$


In the formula, $y\left(r^{\prime}\right)^{\text {represents multidimensional objective function and }} r^{\prime} \in p^{i}{ }^{i}$ is decision variable. According to the above content analysis, assuming that there are $a$ vectors $O_{1}, O_{2}, \cdots, O_{a}$ in the calculation process, randomly select a particle to check its corresponding coding, carry out cyclic iteration, and identify multiple factors mentioned above. If the corresponding value of the factor represented by $r_{a}$ is 1 , then add the factor to $o_{1}$, if the corresponding value is equal to 2, then add the element to ${ }_{O_{2}}$, and so on in order Row derivation placement, arranging the components of $n$ vectors in the above order.

Considering that the fitness value calculated by each factor is different, the fitness function with smaller value is in a disadvantageous position. Therefore, the balance coefficient $\partial$ is introduced for processing, and the balance coefficient is determined by the analysis of the value magnitude of the function in the actual calculation example. Then the calculation formula of multi objective fitness function for railway transportation is as follows:

$$
\text { fitness }\left(r_{a}\right)=\partial^{*} y_{n}\left(r^{\prime}\right)
$$

Through the above calculation, the fitness function corresponding to the particle is obtained, but the particle has different advantages and disadvantages. For the poor particles, help them get rid of the bad position as soon as possible, and speed up the development to other areas; for the high-quality particles, strengthen the development of their corresponding areas, and excavate better solutions. The fitness function is adjusted by the mean fitness of particle population. The mean value of population fitness is calculated as follows:

$$
\text { fitness }\left(x_{i}\right)=\frac{\sum_{i=1}^{n} y\left(r^{\prime}\right)_{a}}{q}
$$

In the formula, $q$ represents the population size, $a$ is a constant and represents the number of key factors. If the result is fitness $\left[r_{a}\right]>$ fitness $\left(r_{q}\right)$, it means that the particle is in a better position, close to the optimal solution, so reduce the search step size [10] of this part of particles, avoid the step size is too large, and miss the optimal solution, adjust the step size by reducing the speed limit of the increase of inertia weight. The formula is as follows: 


$$
\left\{\begin{array}{l}
\text { fitness }\left(r_{a}\right)=\left(\mathfrak{J}_{\text {max }}-\mathfrak{J}\right) * \frac{\text { fitness }\left(r_{a}\right)}{\text { fitness }\left(r_{q}\right)} * S_{\text {max }} \\
S_{\text {max }}=1+\frac{1}{S_{0}}
\end{array}\right.
$$

If fitness $\left[r_{a}\right]<$ fitness $\left(r_{q}\right)$, it shows that the particle is in a worse position and far from the optimal solution. By increasing the search step size of the particle, the search is avoided to be too slow. The method of adjusting step size is to increase inertia weight and reduce speed limitation. The formula is as follows:

$$
\left\{\begin{array}{l}
\text { fitness }\left(r_{a}\right)=\left(\mathfrak{J}_{\min }+\mathfrak{J}\right) * \frac{\text { fitness }\left(r_{a}\right)}{\text { fitness }\left(r_{q}\right)} * S_{\max } \\
S_{\max }=1-\frac{1}{S_{0}}
\end{array}\right.
$$

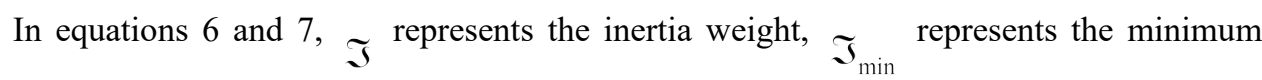
inertia weight, $\mathfrak{I}_{\max }$ represents the maximum inertia weight, $S_{0}$ represents the initial velocity of particles, and $S_{\max }$ represents the maximum velocity of particles. Through the above process, the optimal fitness function is calculated, and the optimal solution of railway logistics is obtained by using this function. The above formula is used as the multi objective optimization model of railway transportation logistics network.

\section{Experimental demonstration and analysis}

Through the above part, the construction process of the multi objective optimization model of railway transportation logistics network based on the improved ant colony algorithm is completed. In order to ensure that the design model in this paper has the ability to optimize the railway transportation network, the use effect of the design model in this paper is obtained by means of comparison.

\subsection{Design of experimental platform}

In order to ensure the consistency of the experimental process and the authenticity of the experimental results, the experimental platform is set to transmit and control the experimental process.

Hardware environment configuration: 
Gddr7 SSD, 1556mhz graphics card, embedded CPU, dual power socket, LED LCD.

Software environment configuration:

The experiment uses SQL 2016 software to complete data storage, $\mathrm{C}++$ programming language, and 32-bit windows 8.0 operating system. The specific experimental environment is as follows.

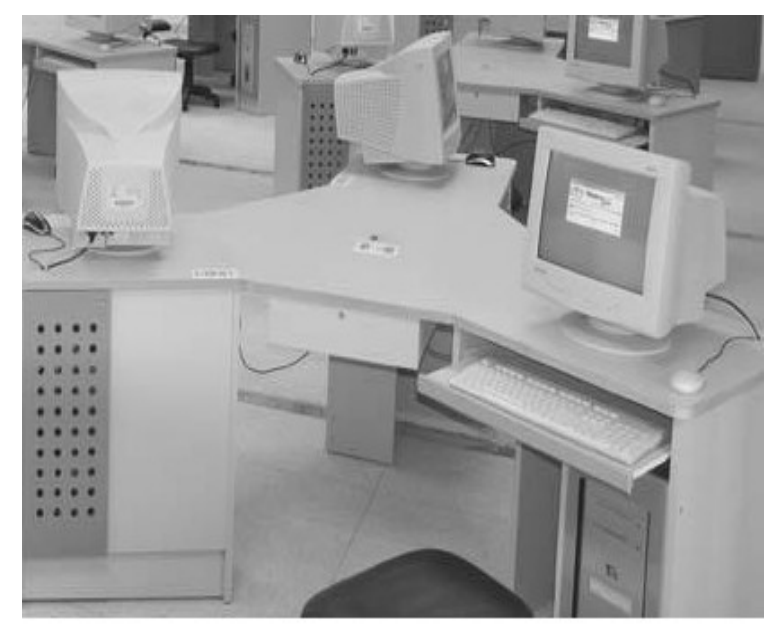

Fig. 4. Experimental environment

Using the above hardware and software to build the experimental environment, the experimental object is set as the network transportation cost of the optimized model and the original optimized model.

In this experiment, we use the form of railway transportation logistics to complete the experimental analysis process. It is set that there are 20 railway logistics points in a certain area, each of which has a construction cost of 100000 yuan and a railway transportation cost of 500 yuan per kilometer. The original model and the optimization model are used to develop the logistics network design, and the optimal route is obtained. The cost calculation is carried out for the design results of the two models, and the cost differences are compared.

\subsection{Analysis of experimental results}

Through the above design, the experimental process is completed, and the specific experimental results are as follows. 


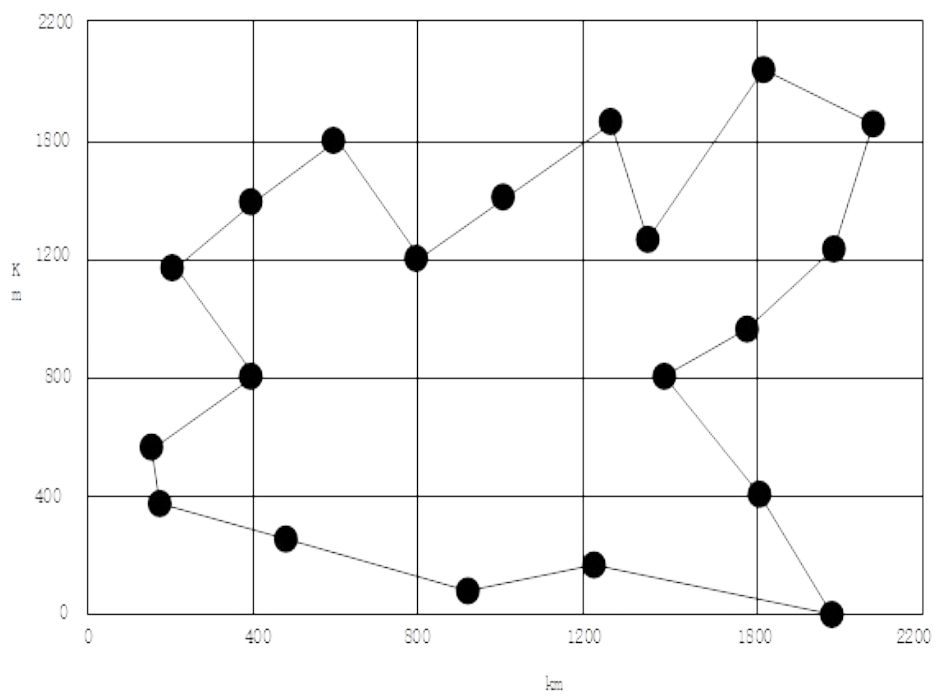

(a) Design results of original model logistics network

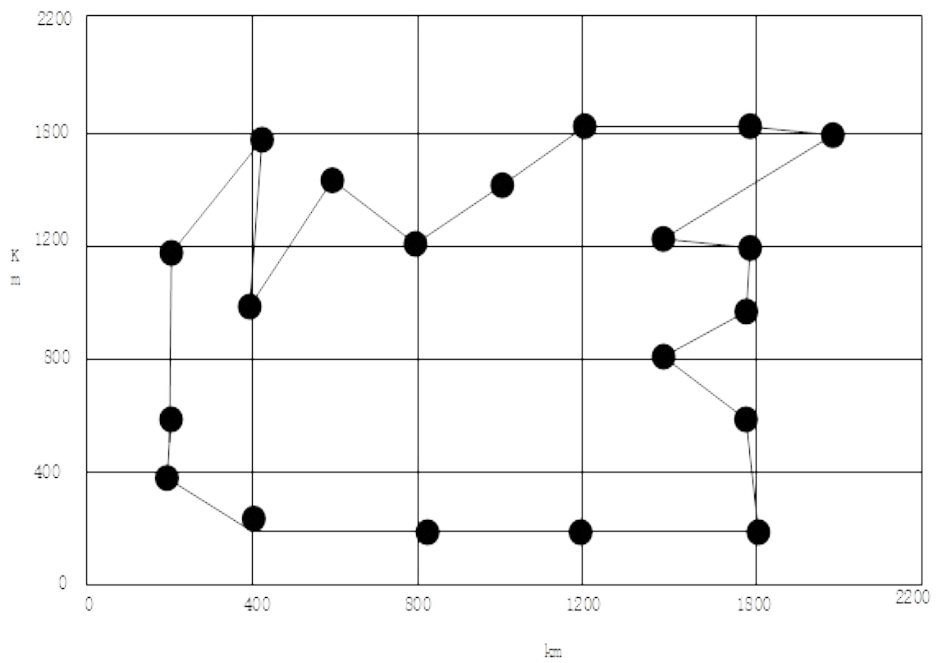

(b) The result of logistics network design of design optimization model in this paper

Fig. 5. Comparison of experimental results

Through the above experimental results, we can see that the logistics network designed by the optimization model is better than that designed by the original model. The logistics network node location designed by the optimization model in this paper is more representative and more scientific. The original model is more random in the location of logistics network nodes, which causes the problem of increasing logistics transportation distance. It is known 
that for each kilometer increase, the logistics transportation cost increases by 500 yuan, and the transportation cost of the original model design result increases by 500 kilometers compared with the design optimization model design result in this paper, then the transportation cost of the original model design result increases by 250000 yuan compared with the design model in this paper. Using the original model results in a significant increase in transportation costs. In conclusion, the optimization effect of the design optimization model in this paper is better. Using the design model in this paper can effectively reduce the transportation costs of the railway transportation network.

\section{Conclusion}

The multi objective optimization problem generally exists in the railway transportation logistics network. In this paper, the multi objective optimization problem in this field is studied. Based on the theory of supply chain management, logistics planning and design, the research system of railway logistics network optimization is summarized. The basic problem model of railway transportation logistics network multi objective optimization is constructed; the logistics path algorithm of improved ant colony algorithm is designed, and the multi objective optimization problem model is solved by combining particle swarm algorithm. The effectiveness and feasibility of the algorithm are verified by case simulation. The results show that the model and algorithm are in line with the reality of the development of logistics enterprises and play an important role in reducing the cost of railway logistics transportation.

\section{Reference}

[1] Wu Xueli ,Jia Yuncong, Zhang Jianhua, et al.: Simulation study of UAV conflict resolution based on an improved ant colony algorithm. Journal of Hebei University of Science and Technology, Vol. 39, no. 8 , pp. 166-175 (2018)

[2] Shen Jiquan, Luo Changwei, Hou Zhanwie, et al.: Service Composition and Optimization Method Based on Improved Ant Colony Optimization Algorithm. Computer Engineering, Vol. 44, no. 12, pp. 6873 (2018)

[3] Cui Chengshuang,Ye Peng.: Dynamic Power Grid Frequency Defense Strategy Based on Ant Colony Algorithm. Journal of Shenyang Institute of Engineering(Natural Science) , Vol. 15, no. 1, pp. 73-77

[4] Shi Xianpeng, Xie Fangyu, Zhang Botao.: A global approach to manned submersibles based on improved ant colony optimization algorithm. The Ocean Engineering, Vol. 37, no. 3, pp. 86-94 (2019) [5]Zhou Jingdong, Zheng Xiaoxuan.: Path Planning Method Based on Improved Ant Colony Algorithm. Journal of Hubei University of Technology, Vol. 33, no. 5, pp. 49-52+101 (2018) 
[6]Zhang Qinghua, Lv Xiaodan.: Research on vehicle routing problem with return and replacement in ecommerce environment and its solution to ant colony algorithm. Computer Engineering and Applications, Vol. 54, no. 22, pp. 239-245 (2018)

[7]Zheng Benli, Li Yuehui.: Study on SDN Network Load Balancing Based on IACO. Computer Science, Vol. 46, no. S1, pp. 291-294 (2019)

[8]Wang Lin, Wang Jin.: Task Scheduling Optimization of Storm Based on Improved Ant Colony Algorithm. Computer Measurement \& Control, Vol. 27, no. 8, pp. 236-240 (2019)

[9]Xu Chenlu, Yang Bin, Zhu Xiaolin.: Research on reverse logistics network design based on robust optimization. Journal of Guangxi University（Natural Science Edition），Vol. 43, no. 6, pp. 2266-2275 (2018)

[10]Pan Zedong, Liu Fuxian.: Simulation of Optimization for Force Maneuver in Battlefield Based on Ant Colony Algorithm. Computer Simulation, Vol. 35, no. 5, pp. 27-30 (2018) 